\title{
Análise fatorial confirmatória do Questionário de Valores Psicossociais - QVP24
}

\author{
Cícero Pereira \\ Universidade Católica de Goiás \\ Leoncio Camino \\ Joseli Bastos da Costa \\ Universidade Federal da Paraíba
}

\begin{abstract}
Resumo
Este artigo apresenta o Questionário de Valores Psicossociais (QVP) como instrumento adequado para a análise dos sistemas de valores de estudantes universitários. No estudo realizado $(\mathrm{N}=300)$, os resultados de uma análise fatorial confirmatória mostram a adequação conceitual da estrutura e dos conteúdos dos quatro sistemas responsáveis pela organização dos valores dos universitários: materialista, religioso, hedonista e pós-materialista. Os resultados também mostram que os sistemas são correlacionados positivamente. A discussão enfatiza a natureza societal dos sistemas de valores e a adequação do $Q V P$ para mensurar esses sistemas.
\end{abstract}

Palavras-chave: valores; sistemas de valores; questionário de valores psicossociais

\begin{abstract}
Confirmatory factorial analysis of the Psychosocial Values Questionnaire - QVP24. This study shows that the Psychosocial Values Questionnaire is an adequate instrument to measure value systems of university students. The results of a Confirmatory Factor Analysis $(\mathrm{N}=300)$ show the conceptual validity of the structure and content that organizes students' value systems: materialist, religious, hedonist and postmaterialist. The results also confirm that these value systems are positively correlated. The discussion emphasizes the societal nature of the values systems and the validity of the Psychosocial Values Questionnaire to measure these systems.
\end{abstract}

Keywords: values; values systems; psychosocial values survey

$\mathrm{N}$ as ultimas décadas, o estudo dos valores sociais tem despertado o interesse de vários pesquisadores. Esse interesse tem-se fundamentado na idéia de que os valores que os indivíduos priorizam são centrais na organização dos sistemas de crenças sociais (Rokeach, 1979a) e dos grupos em sociedade (Williams, 1979). De fato, eles são centrais à compreensão dos comportamentos desses grupos frente aos diversos problemas sociais (Rokeach, 1973; Seligman \& Katz, 1996). A literatura sobre esse tema revela a existência de várias abordagens teóricas e metodológicas sobre a natureza dos valores (Inglehart, 1977; Schwartz, 1992). No Brasil, especificamente, tem sido desenvolvida uma abordagem societal sobre os sistemas de valores e um instrumento de medida para esses sistemas, o Questionário de Valores Psicossociais $(Q V P)$. Esse instrumento tem sido validado em varias investigações (Pereira, Lima, \& Camino, 2001; Pereira, Torres, \& Barros, 2004). A sua elaboração é baseada em um modelo teórico concernente à natureza da estrutura e dos conteúdos dos sistemas de valores dos grupos sociais. Contudo, os estudos sobre a validade desse instrumento utilizam técnicas estatísticas que não permitem testar o modelo teórico subjacente ao $Q V P$, o que, em tese, poderia comprometer a sua validade teórica. Portanto, este artigo apresenta uma investigação que testa empiricamente a adequação do $Q V P$ ao modelo teórico que ele representa.

\section{O Questionário de Valores Psicossociais}

Schwartz (1992) identifica dimensões motivacionais implícitas no modo como os indivíduos e as culturas organizam seus valores. Para esse autor, os valores são representantes cognitivos de um conjunto de necessidades universais na natureza humana. Eles são metas individuais transituacionais que expressam interesses concernentes um tipo motivacional. Schwartz também apresenta o Schwartz's Value Surveys (SVS) para medir dez tipos motivacionais: poder, realização, hedonismo, estimulação, autodireção, universalismo, bene- 
volência, tradição, conformidade e segurança. Os tipos motivacionais se organizam em função de duas dimensões que traduzem relações de compatibilidade e conflitos entre os valores (Schwartz, 1994). A primeira dimensão, formada pela oposição entre a autotranscendência (universalismo e benevolência) e autopromoção (poder e realização), expressa o conflito entre a promoção do bem-estar coletivo e a busca do sucesso pessoal; a segunda dimensão, formada pela oposição entre a abertura à mudança (autodireção, estimulação e hedonismo) e a conservação (segurança, tradição e conformidade), expressa o conflito entre a valorização da mudança e a manutenção do status quo.

Embora tenham sido verificados em várias culturas, a quantidade de tipos motivacionais necessária para organizar os valores mostra-se variável em função do desenvolvimento cognitivo dos indivíduos (Menezes \& Campos, 1997). No Brasil, Tamayo e Schwartz (1993) revelam que oito tipos motivacionais são suficientes para organizar os valores de professores do ensino médio, enquanto nove tipos classificam os valores de estudantes universitários. Em outra investigação realizada no Brasil, Pereira (2000) constata que universitários paraibanos organizam os valores do SVS em apenas quatro tipos motivacionais. Além disso, os valores não se organizam em função de conflitos fundamentais entre tipos motivacionais, mas apenas com base em relações de compatibilidade (Gouveia, Martínez, Meira, \& Milfont, 2001; Pereira, Camino, \& Da Costa, 2003).

As insatisfações com relação ao reducionismo psicológico da abordagem desenvolvida por Schwartz (1992) têm levado Pereira e colaboradores (2001) a desenvolverem uma abordagem que permite realizar interpretações menos psicologizantes dos sistemas de valores e, ao mesmo tempo, elucidar a natureza desses sistemas. Nesse sentido, esses autores propõem uma articulação entre elementos da teoria dos tipos motivacionais com a perspectiva sociológica de Inglehart (1977) sobre os valores materialistas e pós-materialistas. Para Inglehart (1991), os valores representam, sobretudo, mudanças culturais ocorridas na história das sociedades. Inglehart (1994) defende que os valores materialistas surgiram através da mudança do sistema feudal ao sistema de produção capitalista. Do mesmo modo, a estabilidade econômica de algumas sociedades pós-industriais viabilizou a emergência de metas pós-materialistas. Neste sentido, as sociedades que ainda não solucionaram problemas sociais básicos dão mais importância às metas materialistas, enquanto as sociedades que atingiram certo grau de resolução desses problemas valorizam as metas pós-materialistas (Inglehart, 1991). Em estudos realizados em diversas culturas (Bean \& Papadakis, 1994; Braithwaite, Makkai, \& Pittelkow, 1996; Flanagan, 1987; Kidd \& Lee, 1997; Vala, 1994) os valores materialistas foram associados a preocupações concernentes à estabilidade econômica, enquanto os valores pós-materialistas relacionaramse ao bem-estar social, à realização profissional e ao bemestar individual (Pereira \& Camino, 1999).

Para que o desenvolvimento de uma perspectiva societal dos valores seja viável, Da Costa (2000) define os sistemas de valores como estruturas de conhecimento socialmente ela- boradas para sintetizar os elementos de um sistema simbólico amplamente compartilhado. Nessa abordagem, os sistemas de valores expressam os conflitos ideológicos que ocorrem nas sociedades, orientam os comportamentos e estão ancorados nas identidades dos grupos sociais e nos posicionamentos ideológicos derivados dessas identidades. A emergência de um conjunto de valores em uma determinada sociedade está condicionada, não ao fato deles representarem cognitivamente as metas individuais dos membros dessa sociedade, mas pela característica própria dos valores de representarem as diversas tendências ideológicas com as quais os grupos sociais se identificam. Diversas investigações têm sido realizadas com base nesses pressupostos. Seus objetivos são desenvolver o $Q V P$ para avaliar os sistemas de valores e sua relação com as atitudes ideológicas dos grupos sociais (Lima \& Camino, 1995; Pereira et al., 2001).

Na primeira investigação, Torres (1992) apresentou uma escala contendo nove valores $(Q V P-9)$ a estudantes universitários que atribuíram notas, variando entre um e dez, aos valores em função de sua importância para a construção de uma sociedade ideal. Essa autora constatou que os valores da escala configuram-se em dois sistemas: democrático e autoritário. No segundo estudo, Lima e Camino (1995) acrescentaram oito novos valores à versão anterior da escala, formando o $Q V P-17$. Os seus resultados revelaram cinco sistemas subjacentes à adesão aos valores: bem-estar individual, bem-estar econômico, religioso, igualitário e libertário. Finalmente, Pereira, Lima, e Camino (1997) acrescentaram mais oito valores à escala, definindo o $Q V P-25$, e aplicaram-na a estudantes universitários. Esses autores confirmaram a existência de cinco sistemas: bem-estar social; bem-estar individual; bem-estar profissional; religioso; bem-estar econômico.

Considerando que as técnicas de análise de dados empregadas nesses estudos não permitem avaliar as dimensões organizadoras desses sistemas, Pereira et al. (2001) realizaram duas novas pesquisas aplicando o $Q V P-25$. Os resultados de uma análise de aglomerados e de um escalamento multidimensional indicam uma configuração composta por três sistemas de valores: o materialista, o religioso e o pós-materialista, constituído por três subsistemas: bem-estar social; bem-estar individual; bem-estar profissional. Os coeficientes de fidedignidade dos sistemas são elevados nos dois estudos (alfas variando de 0,74 a 0,92). Posteriormente, Pereira, Camino, Da Costa, Lima, Lhullier, e Sandoval (2001) apresentaram duas novas investigações em amostras de universitários da região sul do Brasil, constatando uma organização dos valores igual à verificada nos estudos realizados na Paraíba. Os coeficientes de fidedignidade dos sistemas na região sul também foram elevados (alfas variando de 0,79 a 0,92 ).

Posteriormente, Pereira, Camino, e Da Costa (2003) apresentaram a versão final do $Q V P$ (o $Q V P-24)$, ao inserir na escala valores relacionados a um hipotético sistema hedonista, presente em diversas culturas (Schwartz, 1994). Ao aplicar o QVP-24 em uma amostra de estudantes universitários paraibanos, Pereira et al. (2003) verificaram que esses estudantes organizam seus valores com base em quatro sis- 
temas: materialista, religioso, hedonista e pós-materialista. $\mathrm{O}$ sistema pós-materialista é constituído por três subsistemas: bem-estar individual; bem-estar social; bem-estar profissional. Para testar a convergência entre aos sistemas de valores avaliados com o $Q V P$ e os tipos motivacionais da teoria de Schwartz (1992), Pereira et al. (2003) correlacionaram os sistemas avaliados com o $Q V P$ e os tipos motivacionais avaliados com o $S V S$. O sistema religioso se correlacionou com o tipo motivacional da conservação, pois ambos traduzem um estilo de vida estruturado em torno da harmonia social e da manutenção do status quo (Schwartz, 1994). O sistema materialista se correlacionou com o tipo motivacional da autopromoção, sobretudo com os valores que descrevem o poder social. $\mathrm{O}$ conteúdo do sistema pós-materialista correlacionou-se mais fortemente com os valores do universalismo e da benevolência, ambos indicadores da autotranscendência.

Além disso, os resultados constatados por Pereira et al. (2003) mostram um aspecto peculiar aos valores, também constatado em outras investigações (Gouveia et al., 2001; Lima, 1997; Pereira et al., 2001). Essa peculiaridade refere-se ao fato dos sistemas de valores apresentarem apenas correlações positivas entre si, indicando que os valores são organizados não em função de relações de conflito, mas por suas possíveis compatibilidades. Segundo Pereira et al. (2001), essas correlações mostram que uma concepção sobre valores baseada em relações de conflito (Schwartz, 1996) contradiz a existência da desejabilidade social presente nesses valores.
De fato, as sociedades modernas enfatizam a centralidade dos valores pós-materialistas para o bom desenvolvimento das relações sociais. Paradoxalmente, essa sociedade subordina-se, cada vez mais, às leis do mercado globalizado, visando lucro e acúmulo econômico, que são características do materialismo (Pereira \& Camino, no prelo; Pereira et al., 2003).

Em síntese, os estudos realizados com o QVP-24 mostram sua validade de construto para a avaliação dos sistemas de valores de estudantes universitários. A confiabilidade do instrumento também é elevada, pois, levando-se em conta todos os estudos realizados com o $Q V P$, os coeficientes alfa aplicados aos sistemas apresentam índices de fidedignidade que variam de 0,70 a 0,92 (Média $=0,82 ; D P=0,06$ ). O conjunto de investigações realizadas sobre o $Q V P-24$ permite a elaboração de um modelo psicossocial responsável pela adesão dos grupos sociais aos diversos valores. Esse modelo (Figura 1) é formado por seis construtos e vinte e quatro variáveis observáveis. Os construtos são os sistemas de valores (materialista, religioso, hedonista e pós-materialista) e os subsistemas de valores que representam o sistema pós-materialista (bem-estar individual, bem estar social e bem-estar profissional). Esse modelo, baseado nas interpretações elaboradas por Pereira e colaboradores (2001, 2003), também pressupõe que todas as correlações entre os sistemas são positivas. Isto significa que a organização dos valores não representa conflitos entre metas individuais, mas indicam possíveis relações de compatibilidades entre os valores.

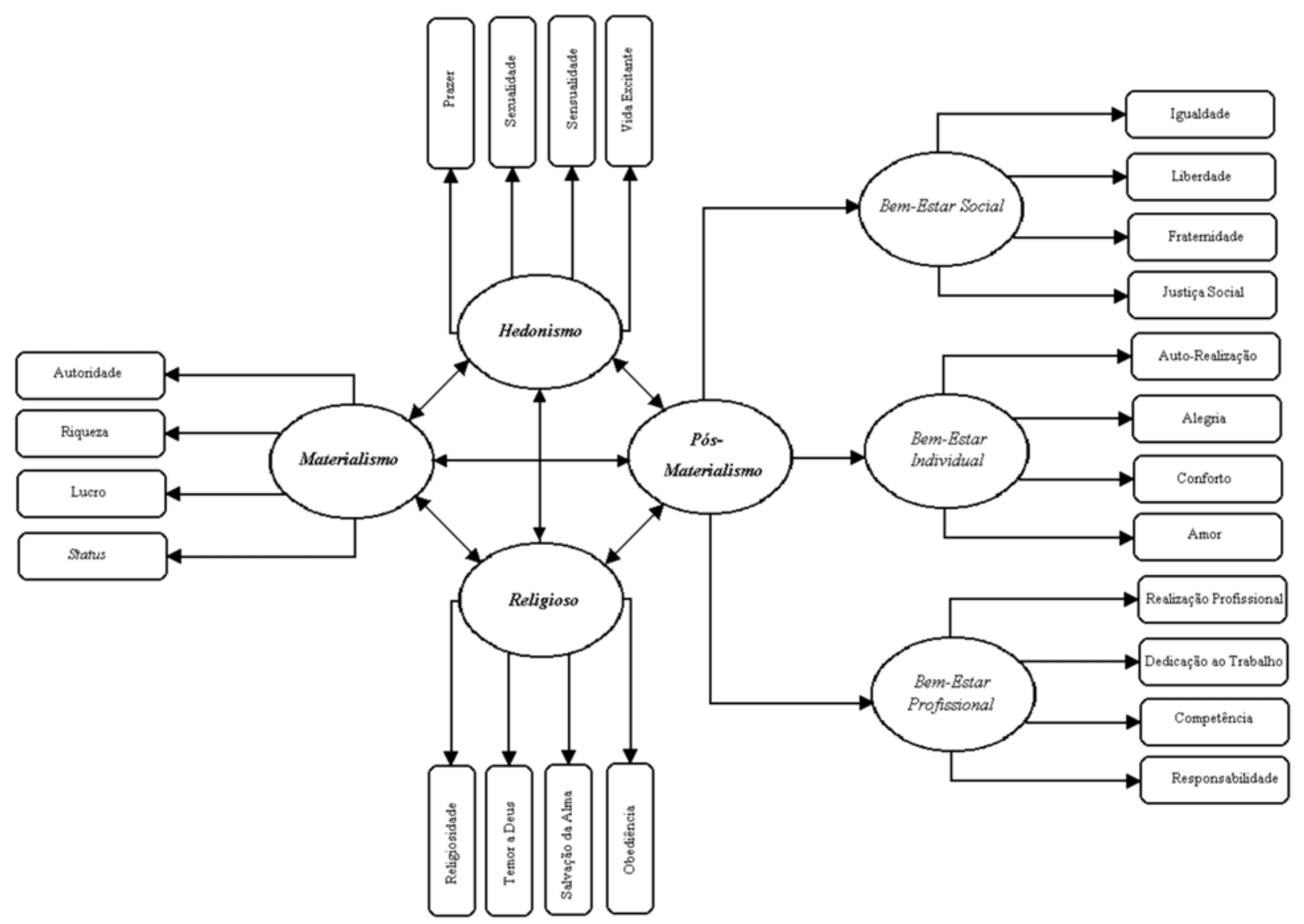

Figura 1. Estrutura e conteúdos dos sistemas de valores. 
Nesse sentido, os valores do $Q V P-24$ parecem descrever de forma adequada o conteúdo dos sistemas e subsistemas do modelo. Entretanto, os estudos realizados com esse instrumento utilizam estatísticas baseadas em matrizes de similaridades e dissimilaridades, como a MDS e a Análise de Aglomerados, que não permitem testar o modelo proposto. Com a finalidade de superar essa fragilidade, este artigo apresenta uma investigação orientada por dois objetivos: (1) confirmar, através de uma análise fatorial confirmatória, a estrutura proposta para a análise dos sistemas de valores de universitários paraibanos; (2) testar a hipótese de que esses sistemas, considerados como estruturas de conhecimento socialmente organizadas, não apresentam relações de oposição, mas apenas associações de compatibilidade entre si. Espera-se que o modelo constituído pelos quatro sistemas de valores seja adequado para descrever a configuração da estrutura e do conteúdo dos valores dos estudantes. Espera-se também que esses sistemas apresentem apenas correlações positivas entre si, mostrando que não há oposição motivacional entre sistemas de valores (Lima, 1997; Pereira et al., 2001).

\section{Método}

\section{Participantes}

Participa desta pesquisa uma amostra composta por 300 estudantes de ambos os sexos de uma universidade localizada na cidade de João Pessoa. A idade dos participantes varia de 16 a 53 anos (Média $=23 ; D P=5,66$ ).

\section{Instrumento}

Os dados foram coletados com o $Q V P-24$. Esse instrumento é constituído por uma lista contendo 24 valores elaborados para avaliar quatro sistemas de valores (Figura 1): materialista (autoridade, lucro, riqueza e status); hedonista (prazer, sensualidade, sexualidade e uma vida excitante); religioso (obediência às leis de Deus; religiosidade, salvação da alma e temor a Deus); pós-materialista, composto por três subsistemas: bem-estar social (fraternidade, igualdade, justiça social e liberdade), bemestar individual (alegria, amor, auto-realização e conforto) e bem-estar profissional (competência, dedicação ao trabalho, realização profissional e responsabilidade). Os estudantes atribuíram uma nota, variando de 1 (sem importância) a 5 (extremamente importante), a cada valor considerando sua importância para a construção de uma sociedade ideal.

\section{Procedimentos}

Os estudantes responderam os questionários em salas de aulas definidas através de sorteios. Para a realização dos sorteios, solicitou-se a grade curricular aos coordenadores dos cursos, contendo os horários de todas as disciplinas de cada curso. Foram sorteadas disciplinas de início, meio e final de curso. Em seguida, solicitou-se aos professores das disciplinas a permissão para as aplicações dos questionários. Os participantes utilizaram entre 10 e 15 minutos para completar o questionário.

\section{Resultados}

Para avaliar a adequação da organização dos conteúdos dos sistemas de valores à estrutura proposta, aplicou-se uma análise fatorial confirmatória através do programa EQS 5,5 para Windows (Bentler, 1995; Bentler \& Wu, 1993). Trata-se de um tipo específico de modelos de equações estruturais (Ullman, 1996). Equações estruturais são um conjunto de técnicas estatísticas que avalia relações simultâneas entre uma ou mais variáveis independentes e uma ou mais variáveis dependentes, permitindo o teste empírico de modelos teóricos (Bentler, 1980; Bonett \& Bentler, 1983). Segundo Bollen (1989), as variáveis inclusas no modelo podem ser tanto construtos psicossociais (os sistemas de valores) quanto variáveis mensuráveis (a adesão a cada valor do questionário). Dois conjuntos de resultados em uma análise fatorial confirmatória devem ser observados (Hu, Bentler, \& Kano, 1992): o ajustamento global do modelo fatorial hipotetizado; a estimação da magnitude do efeito dos construtos sobre as variáveis mensuradas.

Para avaliar a adequação do modelo, o programa calcula o $p$ associado ao valor do $\chi^{2}$, em que a não significância indica a boa adequação do modelo (Bentler \& Bonett, 1980). Outro indicador de adequação é a razão entre o valor de $\chi^{2}$ e os graus de liberdade $\left(\chi^{2} / g l\right)$. Sua interpretação não é consensual entre os autores. As interpretações mais liberais adotam a razão $\chi^{2 / g l}$ menor que cinco como indicativo de bom ajuste do modelo (Garcia \& Sánchez, 1992). As análises mais conservadoras consideram coeficientes menores que dois como critério de adequação do modelo (Ullman, 1996). Contudo, as interpretações mais adequadas são as que adotam índices entre dois e três como indicadores de adequação de modelos teóricos aos dados observados (Kline, 1994). Mesmo assim, por serem sensíveis ao tamanho da amostra, os índices que se baseiam no $\chi^{2}$ podem indicar inadequação do modelo mesmo quando ele for adequado (Bollen, 1986; Kline, 1994). Para superar essa limitação, outros índices de ajustamento (fit), menos sensíveis ao tamanho da amostra são desenvolvidos. Dentre eles, podem ser destacados, o Comparative Fit Index - CFI (Bentler, 1988), o Goodness-ofFit-Index - GFI, juntamente com o Adjusted Goodness-ofFit-Index - AGFI (Jöreskog \& Sörbom, 1989), e o Root Mean Square Error of Aproximation - RMSEA (Browne \& Cudeck, 1993). O modelo será adequado se o $C F I$, o $G F I$ e o $A G F I$ forem superiores a 0,90 (Kline, 1994), ou ainda se o RMSEA apresentar coeficiente inferior a 0,05 (Browne \& Cudeck, 1993).

A estimação dos efeitos das relações hipotetizadas é realizada com base na matriz de covariâncias entre as variáveis. Neste estudo, essa estimação foi realizada livremente através do método da máxima verossimilhança escala. Este é o melhor método em estudos realizados em amostras inferiores a 500 sujeitos e quando a distribuição das variáveis na população pode não ser normal (Hu et al., 1992; Ulleman, 1996), tal como o estudo dos sistemas de valores (Pereira, 2000). De fato, uma das principais características dos valores é a sua alta desejabilidade social (Pereira et al., 2001). As variáveis com maior número de dados ausentes (ambas com n = 3) são status, 
uma vida excitante e prazer. Seguindo os procedimentos estabelecidos por Tabachnick e Fidell (1996), os dados ausentes foram substituídos pela média de sua respectiva variável. Os dados não apresentam outliers univariados ou multivariados. Finalmente, as saturações de um construto (o sistema pósmaterialista) sobre outros construtos (os subsistemas de valores do bem-estar social, individual e profissional) são representadas pela letra grega Gamma $(\gamma)$. As saturações dos valores preditos pelos sistemas são representadas pelo Lambda $(\lambda)$, enquanto as correlações entre os sistemas são simbolizadas pelo $P h i(\phi)$. Também são apresentados os erros de estimação dos construtos (os Distúrbios - D) e dos valores (os Erros $-E$ ).

Com relação ao ajustamento do modelo fatorial proposto, os resultados (Figura 2) indicam que esse modelo é adequado para analisar os valores que servem de base para construção de uma sociedade ideal $\left(\chi_{237}^{2}=570,11\right.$; $p<0,001 ; \chi^{2} / g l=2,4 ; C F I=0,98 ; G F I=0,96 ; A G F I=0,95$; $R M S E A=0,03)$. Como pode ser constatado, o sistema pós- materialista é constituído pelos subsistemas do bem-estar social $(\gamma=0,45)$, do bem-estar individual $(\gamma=1,00)$ e do bem-estar profissional $(\gamma=0,83)$. Os valores igualdade, liberdade, fraternidade e justiça social apresentam saturações elevadas no subsistema bem-estar social ( $\lambda$ variando de 0,31 a 0,69$)$. O subsistema do bem-estar individual organiza a auto-realização, a alegria, ao conforto e ao amor ( $\lambda$ variando de 0,40 a 0,58 ). $O$ subsistema de valores do bem-estar profissional é formado pelos valores da realização profissional, dedicação ao trabalho, competência e responsabilidade ( $\lambda$ variando de 0,48 a 0,68 ). O sistema materialista organiza os valores autoridade, riqueza, lucro e status ( $\lambda$ variando de 0,41 a 0,69 ). O sistema religioso é indicado pelos valores religiosidade, temor a Deus, salvação da alma e obediência às leis de Deus ( $\lambda$ variando de $0,68$ a 0,84$)$. Os valores prazer, sexualidade, sensualidade e uma vida excitante têm saturação elevada no sistema hedonista ( $\lambda$ variando de 0,59 a 0,75 ). Todas as saturações são estatisticamente significativas, com $p<0,05$.

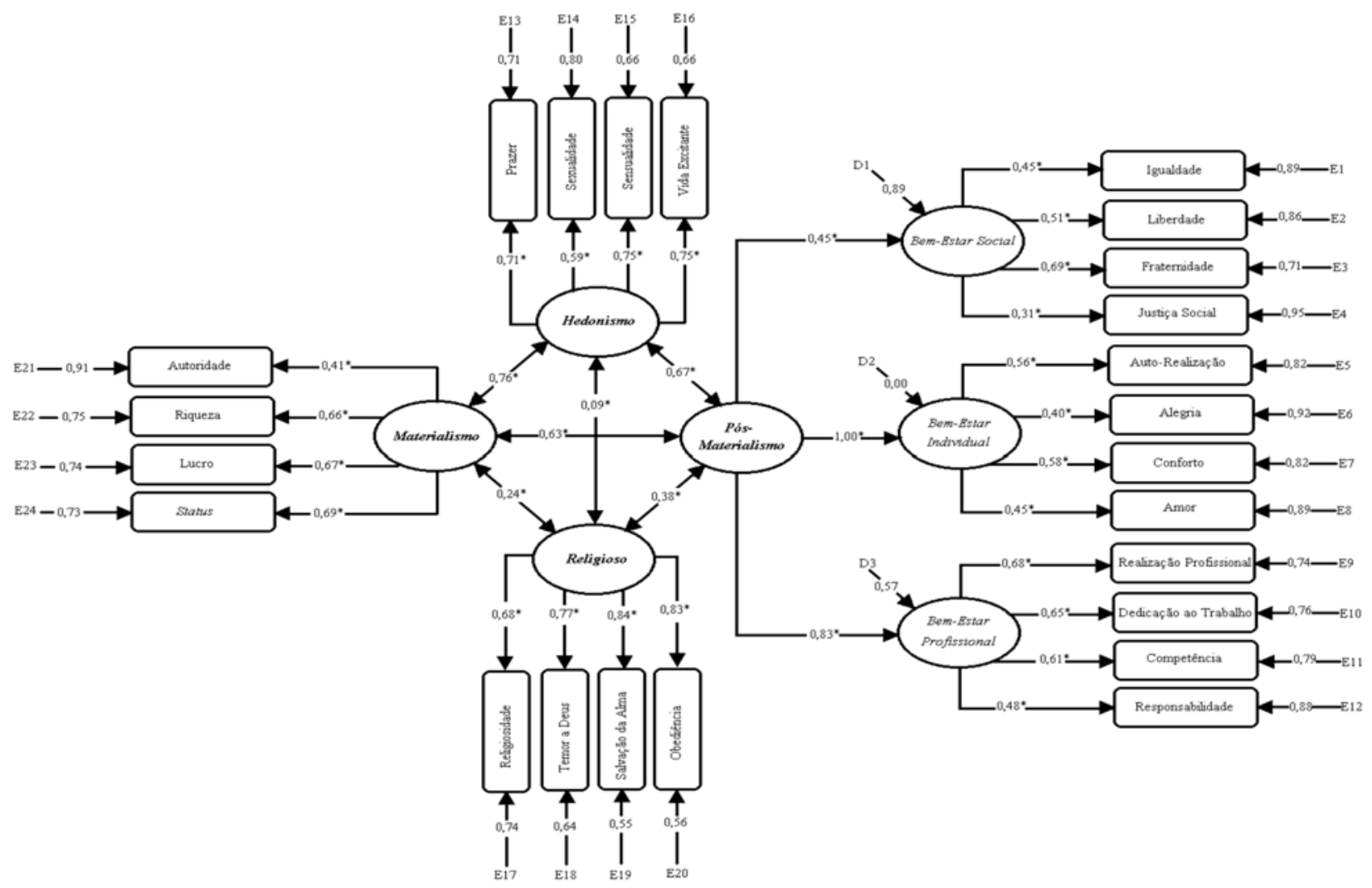

Notas

$D 1-D 3=$ Erro de estimação dos construtos

$E 1-E 24=$ Erros de medida dos valores

Os valores apresentados são coeficientes path padronizados

* $p<0,05$

Figura 2. Resultados da análise fatorial confirmatória aplicada à estrutura e aos conteúdos dos sistemas de valor. 
Com relação à segunda hipótese, a de que não se pode falar em relações de conflito entre os valores, os resultados indicam que, como esperado, as correlações entre os sistemas são positivas e estatisticamente significativas: materialista versus pós-materialista $(\phi=0,63 ; p<0,05)$; materialista versus religioso $(\phi=0,24 ; p<0,05)$; religioso versus pósmaterialista $(\phi=0,38 ; p<0,05)$; hedonista versus pós-materialista $(\phi=0,67 ; p<0,05)$; hedonista versus religioso $(\phi=0,09$; $p<0,05)$; hedonista versus materialista $(\phi=0,76 ; p<0,05)$. Finalmente, a análise da fidedignidade dos sistemas pós-materialista, materialista, religioso e hedonista mostra alfas = 0,$76 ; 0,70 ; 0,87$ e 0,79 , respectivamente, na análise de sua consistência interna.

\section{Discussão}

Como indicado, este artigo é orientado por dois objetivos: (1) confirmar a estrutura proposta para a análise dos sistemas de valores de universitários paraibanos; (2) testar a hipótese de que esses sistemas apresentam apenas relações de compatibilidade entre si. Os resultados da análise fatorial confirmatória mostram que o $Q V P-24$ é um instrumento empiricamente válido para a análise dos sistemas de valores de estudantes universitários. Isto significa que esses estudantes organizam seus valores com base nos quatro sistemas constatados em outras investigações realizadas na Paraíba (Pereira et al., 2001): materialista, religioso, hedonista e pós-materialista. Sugere-se a utilização desse instrumento para a análise dos sistemas de valores dos grupos sociais em outras regiões do país, tal como a investigação realizada por Pereira, Camino et al. (2001) em universitários da região sul.

Os resultados também permitem confirmar a hipótese de que a oposição entre os sistemas de valores é um artefato metodológico produzido pelo tipo de técnica estatística utilizada na análise dos valores, sobretudo as que se baseiam nos princípios das escalas multidimensionais. Em que se fundamenta essa afirmação? Uma reflexão sobre os resultados encontrados neste estudo e os postulados por Schwartz (1992) e Inglehart (1977) sobre a possibilidade de relações de conflito ente valores pode ser útil para que se encontre uma solução plausível para esse questionamento. Existe uma clara distinção entre os resultados deste estudo e os das investigações realizadas por Schwartz (1992). Este autor, por considerar que os valores são derivados de necessidades universais e individuais, admite os tipos motivacionais estão a serviço da satisfação dessas necessidades e, portanto, podem representar compatibilidades ou conflitos axiológicos. Realmente, a figura geométrica produzida pela Smallest Space Analysis (Guttman, 1968), ou pela MDS (Kruskal \& Wish, 1978) organiza a adesão aos valores em lados opostos na figura. Contudo, como mostram os resultados apresentados neste estudo, essa localização não representa oposição real entre valores, pois estatísticas baseadas em medidas de dissimilaridade forçam uma oposição que não se dá na mente das pessoas (Lima, 1997). As correlações positivas entre os sistemas indicam que os valores são truísmos culturais, pois a sua desejabilidade social tem sido a característica mais enfatizada nos estudos sobre valores (Rokeach, 1973; Schwartz, 1996). Esse raciocínio permite afirmar que as pessoas de fato valorizam os valores (Pereira et al., 2001). Nesse sentido, parece ser teoricamente incoerente admitir nos valores a existência de um forte componente de desejabilidade social e a proposta de relações de conflitos entre eles.

Com isso não se pretende afirmar que não haja diferenças entre valores. As diferenças ocorrem tanto no grau de preferência que as sociedades dão aos sistemas de valores quanto nos tipos de contextos em que as preferências são efetivadas. É neste sentido que os valores são crenças (Rokeach, 1979b) ou concepções (Schwartz, 1994) desejáveis socialmente (Deschamps \& Devos, 1993). A partir da concepção de valores como construtos sociais (Pereira et al., 2001), e não como decorrentes de uma hierarquia de necessidades (Schwartz, 1992), não há como conceber incompatibilidade entre sistemas de valores, pois eles são sociais (Beattie, 1980) e amplamente compartilhados no interior dos grupos sociais (Maio \& Olson, 1998). Assim, os sistemas de valores, considerados como estruturas sociais de conhecimentos partilhados, não estão a serviço das hierarquias individuais de necessidades opostas, mas parecem refletir as diversas maneiras que as ideologias sociais encontram para ser justificadas. Essa característica explicita o vinculo dos valores com a política, ao destacar o seu caráter eminentemente ideológico (Billig, 1987).

O debate sobre os resultados deste estudo e os postulados de Inglehart (1991) exige uma análise psicossocial cuidadosa das interpretações propostas por esse autor para uma possível oposição entre valores materialistas e pós-materialistas. Segundo Inglehart (1977), as sociedades com profundos problemas econômicos, de segurança e de organização política valorizam muito o materialismo e pouco o pós-materialismo, enquanto as sociedades que atingiram um determinado grau de resolução desses problemas valorizam metas pósmaterialistas e dão menos importâncias às metas materialistas. Isso explicaria o fato das pesquisas realizadas nos Estados Unidos e na Europa (Inglehart, 1991) mostrarem correlações negativas entre valores pós-materialistas e materialistas. Segundo Pereira et al. (2001), essa relação é peculiar a investigações realizadas em determinados países pós-industriais que, após um período de escassez econômica, conseguiram produzir uma estrutura político-econômica que permite a superação desses problemas com o acúmulo do poder econômico. Embora os valores pós-materialistas sejam freqüentes na maioria das culturas capitalistas ocidentais (Inglehart, 1994; Kidd \& Lee, 1997; Marks, 1997; Van Deth, 1983), nos países em via de desenvolvimento, que não passaram pelo processo de superação da escassez com o acúmulo econômico, os valores materialistas não se contrapõem às metas pós-materialistas (Pereira et al., 2001). Nesses países, a construção de uma sociedade ideal deve passar tanto pelas características do pós-materialismo quanto pelos elementos do materialismo (Pereira \& Camino, 1999). Assim, na representação que os grupos sociais fazem de uma sociedade ideal, podem conviver harmonicamente os valores que descrevem o pós-materialismo com valores ligados à autoridade, à riqueza ao lucro e ao status, os quais descrevem o sistema 
materialista, tal como indicam as correlações positivas verificadas entre esses sistemas.

\section{Referências}

Bean, C., \& Papadakis, E. (1994). Polarized priorities or flexible alternatives? Dimensionality in Inglehart's materialism-posmaterialism scale. International Journal of Public Opinion Reseach,_6, 264-297.

Beattie, J. (1980). Introdução à antropologia social. São Paulo: Companhia Editora Nacional.

Bentler, P. M. (1980). Multivariate analysis with latent variables: causal modeling. Annual Review of Psychology, 31, 419-456.

Bentler, P. M. (1988). Comparative fit indexes in structural models. Psychological Bulletin, 107, 238-246.

Bentler, P. M. (1995). EQS: Structural equations program manual. Encino, Califórnia: Multivariate Software.

Bentler, P. M., \& Bonett, D. G. (1980). Significance tests and goodness of fit in the analysis of covariance structures. Psychological Bulletin, 88, 588-606.

Bentler, P. M., \& Wu, E. J. C. (1993). EQS/Windows user's guide. Los Angeles: BMDP Statistical Software.

Billig, M. (1987). Arguing and thinking: a rhetorical approach to social psychology. Cambridge: Cambridge University Press.

Bollen, K. A. (1986). Sample size and Bentler and Bonett's nonnormed fit index. Psychometrika,_51, 375-377.

Bollen, K. A. (1989). Structural equations with latent variables. Nova York: Wiley.

Bonett, D. G., \& Bentler, P. M. (1983). Goodness-of-fit procedures for evaluation and selection of log-linear models. Psychological Bulletin, 93, 149-166.

Braithwaite, V., Makkai, T., \& Pittelkow, Y. (1996). Inglehart's materialismpostmaterialism concept: clarifying the dimensionality debate through Rokeach's model of social values. Journal of Applied Social Psychology, 26, 1536-1555.

Browne, M. W., \& Cudeck, R. (1993). Alternative ways of assessing model fit. In K. A. Bollen, \& L. S. Long (Orgs.), Testing structural equation models (pp. 136-162). Newbury Park, Califórnia: Sage.

Da Costa, J. B. (2000). Visões sociais de democracia: um estudo psicossociológico dos significados da democracia. Tese de Doutorado não-publicada, Curso de Pós-graduação em Psicologia Social, Pontifícia Universidade Católica de São Paulo, São Paulo.

Deschamps, J., \& Devos, T. (1993). Valeurs, cultures et changement. Intercultures, 1, 17-28.

Flanagan, S. C (1987). Value change in industrial societies. American Political Science Review, 81, 1303-1319.

Garcia, M. A. M., \& Sánchez, J. F. (1992). Análisis confirmatorio de la estructura dimensional de un cuestionario para la evaluación de la calidad de la enseñanza. Investigaciones Psicológicas, 11, 73-82.

Gouveia, V., Martínez, E., Meira, M., \& Milfont, T. L. (2001). A estrutura e o conteúdo universais dos valores humanos: análise fatorial confirmatória da tipologia de Schwartz. Estudos de Psicologia, 6, 133-142.

Guttman, L. (1968). A general no metric technique for finding the smallest coordinate space for a configuration of points. Psychometrica, 33, 469-506.

Hu, L. T., Bentler, P. M., \& Kano, Y. (1992). Can test statistics in covariance structure analysis be trusted? Psychological Bulletin, 112, 351-362.

Inglehart, R. (1977). The silent revolution. Princeton: Princeton University Press.

Inglehart, R. (1991). El cambio cultural en las sociedades industriales avanzadas. Madri: Siglo XXI.

Inglehart, R. (1994). Modernización y post-modernización: la cambiante relación entre el desarrollo económico, cambio cultural y político. In J. D. Nícolas, \& R. Inglehart (Orgs.), Tendencias mundiales de cambio en los valores sociales y políticos (pp. 157-170). Madri: Fundesco.

Jöreskog, K. G., \& Sörbom, D. (1989). LISREL 7: A guide to the program and applications. Mooresville, Indiana: Scientific Software.
Kidd, Q., \& Lee, A. R. (1997). Postmaterialist values and the environment: a critique and reappraisal. Social Science Quarterly, 78, 1-15.

Kline, P. (1994). An easy guide to factor analysis. Londres: Routledge.

Kruskal, J. B., \& Wish, M. (1978). Multidimensional scaling. Londres: Sage.

Lima, M. E. (1997). Valores, participação política, atitudes face a democracia e ao autoritarismo: uma análise da socialização política dos universitários da Paraíba. Dissertação de mestrado não-publicada, Universidade Federal da Paraíba, João Pessoa.

Lima, M. E., \& Camino, L. (1995). A Política na vida de estudantes universitários: uma análise em termos de espaço político e de valores. In M. J. L. Silva (Org.), Iniciados (pp. 11-35). João Pessoa: Editora Universitária.

Maio, G. R., \& Olson, J. M. (1998). Values as truisms: evidence and implications. Journal of Personality and Social Psychology, 74, 294 - 311.

Marks, G. N. (1997). The formation of materialist and pos-materialist values. Social Science Research, 26, 52-68.

Menezes, I., \& Campos, B. (1997). The process of value-meaning construction: a cross-sectional study. European Journal of Social Psychology, 27, 55-77.

Pereira, C. (2000). Representações sociais do envolvimento nos direitos humanos. Dissertação de Mestrado não-publicada, Universidade Federal da Paraíba, João Pessoa.

Pereira, C., \& Camino, L. (1999). Proposta de um modelo psicossociológico para o estudo das atitudes políticas de estudantes universitários. In M. F. V. Souza (Org.), Iniciados (pp. 427-440). João Pessoa: Editora Universitária.

Pereira, C., Camino, L., \& Da Costa, J. (2003). Desenvolvimento de uma abordagem societal para análise dos sistemas de valores. Artigo submetido à publicação na revista Psicologia: Reflexão e Crítica.

Pereira, C., Camino, L., Da Costa, J., Lima, M. E., Lhullier, L., \& Sandoval, S. (2001). Sistemas de valores e atitudes democráticas de estudantes universitários do Sul do Brasil. Estudos, 28, 639-671.

Pereira, C., Lima, M. E., \& Camino, L. (1997). A prática política: uma análise psicossociológica em termos de valores e inserção social. Em M. J. L. Silva (Org.), Iniciados (pp. 199-235). João Pessoa: Editora Universitária.

Pereira, C., Lima, M. E., \& Camino, L. (2001). Sistemas de valores e atitudes democráticas de estudantes universitários de João Pessoa. Psicologia: Reflexão e Crítica, 14, 177-190.

Pereira, C., Torres, A. R. R., \& Barros, T. S. (2004). Sistemas de valores e atitudes democráticas de estudantes universitários. Psicologia: Teoria \& Pesquisa, 20(1), 1-10

Rokeach, M. (1973). The nature of human values. Nova York: Free Press.

Rokeach, M. (1979a). Introduction. Em M. Rokeach (Org.), Understanding human values: individual and societal (pp. 1-11). Nova York: Free Press.

Rokeach, M. (1979b). The two-value model of political ideology and British politics. In M. Rokeach (Org.), Understanding human values: individual and societal (pp. 192-196). Nova York: Free Press.

Schwartz, S. H. (1992). Universals in the content and structure of values: theoretical advanced and empirical testes in 20 countries. In M. Zanna (Org.), Advances in experimental social psychology (Vol. 25, pp. 1-65) Orlando, Flórida: Academic.

Schwartz, S. H. (1994). Are there universal aspects in the structure and contents of human values? Journal of Social Issues, 50, 19-45.

Schwartz, S. H. (1996). Value priorities and behaviour: applying a theory of integrated value systems. In C. Seligman, J. M. Olson, \& M. P. Zanna (Orgs.), The psychology of values: The Ontario Symposium (Vol. 8, pp. 124). Mahwah, New Jersey: Lawrence Erlbaum.

Seligman, C., \& Katz, A. (1996). The dynamics of value systems. In C. Seligman, J. M. Olson, \& M. P. Zanna (Orgs.), The psychology of values: The Ontario Symposium (Vol. 8, pp. 53-75). Mahwah, New Jersey: Lawrence Erlbaum.

Tabachnick, B.G., \& Fidell, L. S. (1996). Using multivariate statistics. Nova York: Harper \& Row. 
Tamayo, A., \& Shwartz, S. H. (1993). Estrutura motivacional dos valores humanos. Psicologia: Teoria e Pesquisa, 9, 328-346.

Torres, A. R. R. (1992). Uma análise psicossocial da identificação partidária: o caso dos estudantes da UFPb nas eleições de 1988, 1989 e 1990. Dissertação de mestrado não-publicada, Universidade Federal da Paraíba, João Pessoa.

Ullman, J. B. (1996). Structural equation modeling. In B. G. Tabachnick, \& L. S. Fidell (Orgs), Using multivariate statistics (pp. 709- 811). Nova York: Harper \& Row.
Vala, J. (1994). La emergencia de los valores post-materialistas en Portugal. In J. D. Nícolas, \& R. Inglehart (Orgs.), Tendencias mundiales de cambio en los valores sociales y políticos (pp. 157-170). Madri: Fundesco.

Van Deth, J. W. (1983). Ranking the ratings: The case of materialist and posmaterialist value orientations. Political Methodology, 9, 407-431.

Williams, R. M. (1979). Change and stability in values and value systems: a sociological perspective. In M. Rokeach (Org.), Understanding human values: individual and societal (pp. 15-46). Nova York: Free Press.

Cícero Pereira, doutorando em Psicologia Social no Instituto Superior de Ciências do Trabalho e da Empresa (Lisboa, Portugal), é professor de Psicologia Social na Universidade Católica de Goiás. Endereço para correspondência: Grupo de Pesquisa em Comportamento Político; Caixa Postal 5069; João Pessoa, PB; CEP 58051-970. E-mail: cicero.psi@ ucg.br

Leoncio Camino, doutor em Psicologia Social pela Université Catholique de Louvain (Bélgica), é professor no Programa de Pós-Graduação em Psicologia Social da Universidade Federal da Paraíba.

Joselí Bastos da Costa, doutor em Psicologia Social pela Pontifícia Universidade Católica de São Paulo, é professor no Programa de Pós-Graduação em Psicologia Social da Universidade Federal da Paraíba e coordenador do Grupo de Pesquisa em Comportamento Político. 